\title{
Predictive factors, microbiology and outcome of patients with parapneumonic effusion
}

\author{
M. Falguera*, J. Carratalà\#, S. Bielsa*, C. García-Vidal”, A. Ruiz-González*, I. Chica*, \\ F. Gudiol ${ }^{\#}$ and J.M. Porcel*
}

ABSTRACT: We aimed to determine the incidence, clinical consequences and microbiological findings related to the presence of pleural effusion in community-acquired pneumonia, and to identify predictive factors for empyema/complicated parapneumonic effusion.

We analysed 4,715 consecutive patients with community-acquired pneumonia from two acute care hospitals. Patients were classified into three groups: no pleural effusion, uncomplicated parapneumonic effusion and empyema/complicated parapneumonic effusion.

A total of $882(19 \%)$ patients had radiological evidence of pleural fluid, of whom $261(30 \%)$ met criteria for empyema/complicated parapneumonic effusion. The most important event related to the presence of uncomplicated parapneumonic effusion was a longer hospital stay. Relevant clinical and microbiological consequences were associated with empyema/complicated parapneumonic effusion. Five independent baseline characteristics could predict the development of empyema/ complicated parapneumonic effusion: age $<60$ yrs $(p=0.012)$, alcoholism $(p=0.002)$, pleuritic pain $(p=0.002)$, tachycardia $>100$ beats $\cdot \mathrm{min}^{-1}(p=0.006)$ and leukocytosis $>15,000 \mathrm{~mm}^{-3}(p<0.001)$. A higher incidence of anaerobes and Gram-positive cocci was found in this subgroup of patients.

We conclude that only the development of empyema/complicated parapneumonic effusion carried relevant consequences; this condition should be suspected in the presence of some baseline characteristics and managed by using antimicrobials active against Gram-positive cocci and anaerobes.

KEYWORDS: Community-acquired pneumonia, empyema, pleural effusion

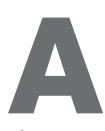

ccording to the radiological picture, patients with community-acquired pneumonia (CAP) can be divided into two groups: those with and those without pleural effusion. Subsequently, the biochemical and microbiological analyses of the pleural fluid and its macroscopic appearance provide criteria for the classification of patients with pleural effusion into two new subgroups: patients with uncomplicated parapneumonic effusion (UPE) and patients with empyema/ complicated parapneumonic effusion (E/CPE) [1]

This classification has important consequences regarding management and patient outcomes. Thus, while those with UPE are treated with antibiotics alone, patients with $\mathrm{E} / \mathrm{CPE}$ require pleural drainage with or without fibrinolytics or thoracic surgery $[2,3]$. In addition, the latter also have a worse prognosis, particularly when the adequate management is delayed [4]. Despite the relevance of this event, few studies have focused on analysing and comparing the characteristics of these subgroups of patients.

In the present study, we aimed to evaluate epidemiological, clinical, aetiological and outcome characteristics of patients with CAP according to the presence or the absence of pleural effusion, and, in the former scenario, according to the characteristics of the fluid. We also searched for predictive factors of $\mathrm{E} / \mathrm{CPE}$ among those patients with pleural effusion.

\section{METHODS \\ Patients}

Patients included in this cohort were recruited from databases developed at two acute-care university hospitals in Spain (the Hospital Arnau de Vilanova, Lleida, and the Hospital de Bellvitge, Barcelona). This study was approved by the scientific and ethics committees of the respective institutions.

Earn CME accreditation by answering questions about this article. You will find these at the back of the printed copy of this issue or online at www.erj.ersjournals.com/misc/cmeinfo.xhtml

AFFILIATIONS

*Internal Medicine, Hospital Universitari Arnau de Vilanova, Universitat de Lleida, Institut de recerca Biomèdia de Lleida (IRBLLEIDA), Lleida, Ciber de Enfermedades Respiratorias (Ciberes), and \# Infectious Diseases Service, Hospital Universitari de Bellvitge, Universitat de Barcelona, Institut d'Investigació Biomèdica de Bellvitge (IDIBELL), Spanish Network for Research in Infectious Diseases (REIPI), Barcelona, Spain.

\section{CORRESPONDENCE}

M. Falguera

Service of Internal Medicine

Hospital Universitari Arnau de

Vilanova

Rovira Roure 80

25198 Lleida

Spain

E-mail: mfalguera@comll.cat

Received:

Jan 012011

Accepted after revision:

April 122011

First published online:

May 122011 
Over a 13-yr period (February 1996 to December 2008), data on all consecutive immunocompetent patients aged $\geqslant 18 \mathrm{yrs}$ and hospitalised with CAP were prospectively and individually collected.

CAP was defined as an acute illness characterised by two or more of the following clinical parameters: fever, chills, cough, sputum production, pleuritic pain and signs of lung consolidation, along with the presence of an infiltrate on the chest radiograph consistent with acute infection.

Exclusion criteria were: 1) infection caused by tuberculosis, fungi or opportunistic microorganisms; 2) primary empyema; 3) immunosuppression including HIV infection, haematological neoplasms, solid-organ and bone-marrow transplantation, neutropenia and immunosuppressive therapies; 4) nosocomial pneumonia; and 5) healthcare-associated pneumonia (for patients recruited before 2005, we excluded those residing in a nursing home or long term-care facility).

\section{Data collection}

The following parameters were evaluated. 1) Epidemiological data: age, sex, smoking habit, alcohol abuse $\left(>60 \mathrm{~g} \cdot \mathrm{day}^{-1}\right)$, risk factors for aspiration other than alcohol [5], prior antibiotic therapy, pneumococcal vaccination and the presence of any underlying disease, such as chronic obstructive pulmonary disease, diabetes mellitus, heart failure, neoplasm, chronic renal insufficiency, chronic liver disease or cerebrovascular disease with significant sequelae. 2) Clinical findings: acute onset of disease, duration of the clinical picture, chills, cough, sputum production, pleuritic pain, signs of pulmonary consolidation (dullness, vocal fremitus, decreased breath sounds, egophony, inspiratory crackles and/or pleural friction rub) and acute mental confusion. 3) Vital signs upon admission: body temperature, respiratory rate, cardiac frequency, systolic and diastolic blood pressures, and oxygen saturation measured by pulse oximetry while breathing ambient air. 4) Blood analyses: serum C-reactive protein, albumin, sodium, urea, white blood cell count, neutrophil count, haematocrit, arterial $\mathrm{pH}$, arterial oxygen tension and arterial carbon dioxide tension, and results of blood cultures. 5) Pneumonia severity index (PSI) and CRB65 $[6,7] .6)$ Outcome parameters: length of hospital stay and 30-day mortality. After discharge, all patients were followed-up until the resolution of the process.

\section{Pleural fluid collection and examination}

At admission, all patients underwent a chest radiograph; thoracic ultrasound was performed when required. A chest radiograph was repeated during the course of disease if there was clinical suspicion of complications. When the presence of pleural fluid was detected, a $20-\mathrm{mL}$ sample was obtained by thoracocentesis, the exception being patients with small pleural effusions thought to be unsafe for pleural aspiration. The sample of fluid was collected under aseptic conditions and processed according to standard procedures. First, the macroscopic aspect of pleural fluid was evaluated. Subsequently, the specimen was inoculated into two culture bottles ( $5 \mathrm{~mL}$ was inoculated into an aerobic bottle and $5 \mathrm{~mL}$ into an anaerobic bottle), and was conventionally processed. The sample was also routinely processed for cytology and, according to the clinical suspicion, cultured for mycobacteria and fungi. Additionally, biochemical pleural fluid analyses, including $\mathrm{pH}$, protein, glucose and lactate dehydrogenase, were performed. This study was not completed for patients with macroscopic evidence of empyema.

\section{Aetiological diagnosis}

Blood, sputum and pleural fluid cultures, urinary antigen detection tests for Streptococcus pneumoniae and Legionella pneumophila, and paired serologies constituted the routine diagnostic tests. Isolation of microorganisms in blood or pleural fluid or seroconversion provided a definitive microbial diagnosis, whereas positive sputum or urinary tests indicated only a probable microbial diagnosis. The following isolates were always considered as contaminants: coagulase-negative staphylococci, Micrococcus spp., Propionibacterium spp. and diphteroids.

\section{Distribution of patients}

Three subsets of patients were established as follows. 1) Patients without pleural effusion. 2) Patients with UPE. In this subset we included patients with pleural effusion that did not meet criteria for E/CPE. 3) Patients with E/CPE. Empyema was defined as either the presence of macroscopic frank pus or a positive Gram stain or culture. Complicated parapneumonic effusion was defined as a nonpurulent fluid that met at least one of the following criteria: $\mathrm{pH}<7.20$, lactate dehydrogenase $>1,000 \mathrm{IU} \cdot \mathrm{L}^{-1}$ or glucose $<60 \mathrm{mg} \cdot \mathrm{dL}^{-1}[8]$.

\section{Statistical analysis}

Data were analysed using the statistical software package SPSS (version 17.0; SPSS Inc., Chicago, IL, USA).

Data of nonparametric distributions are expressed as medians (25-75th quartiles). Parameters of subsets of patients were compared by performing univariate analyses. Between-group comparisons were performed with Kruskal-Wallis test and Mann-Whitney post hoc test for continuous variables, as well as a Chi-squared test with post hoc analysis of adjusted residuals for categorical variables. A two-tailed p-value $<0.05$ indicated statistical significance.

Multivariable analyses were performed to determine factors independently associated with E/CPE, as compared with patients with UPE. Potential predictive factors, including variables found to be significant in the univariate analysis, were entered into a binary logistic regression analysis. To improve the selection process for the logistic regression model, the criterion adapted by HOSMER and LEMESHOW [9] was used. Cases with missing values were excluded from the multivariable analysis. Continuous variables were recategorised into binary factors using the best discriminant cut-off points. Results of multivariable analyses are reported as odds ratios with $95 \%$ confidence intervals and p-values. We also estimated the area under the receiver operating characteristic (ROC) curve as well as the sensitivity, specificity and positive and negative predictive values of the model.

\section{RESULTS}

\section{Study population}

A total of 4,715 cases satisfied the inclusion criteria and were enrolled in the study, of which $882(19 \%)$ had radiological evidence of pleural effusion. E/CPE was diagnosed in 261 patients, which represents $6 \%$ of the overall group of patients with CAP and $30 \%$ of those with parapneumonic effusion. 
Baseline characteristics and outcome parameters of the three groups of patients are shown in table 1 . When patients without pleural fluid and patients with UPE were compared, UPE was associated with acute onset of illness, cough, pleuritic pain, lower albumin level and higher neutrophil count. Attending to outcomes, the development of UPE was related to higher risk of bacteraemia and increased length of hospitalisation. Crude mortality and mortality adjusted for PSI were not associated with UPE.

\section{Predictive factors for $E / C P E$}

Patients with UPE and patients with E/CPE were first compared in a univariate analysis and statistically significant differences were found in numerous baseline characteristics (table 1). Thus, regarding the epidemiological parameters, E/CPE was significantly associated with younger age, smoking and alcohol abuse, and absence of any underlying disease including chronic obstructive pulmonary disease, heart failure, and cerebrovascular disease or pneumococcal vaccination. In relation to clinical manifestations, E/CPE was significantly associated with a longer disease duration, the presence of pleuritic pain and signs of consolidation, and the absence of fever, cough and an acute disease onset. These patients also showed significantly higher pulse and respiratory rates and lower axillary temperature. With regard to the laboratory results, patients with E/CPE showed higher values of white blood cell and neutrophil counts, lower albumin levels and higher bacteraemia rate. Patients with E/CPE were classified into less severe PSI categories; however, they had longer hospital stays and higher mortality rates, both crude and adjusted for PSI.

Baseline characteristics potentially associated with E/CPE were entered into a multivariable analysis, with the exception of the albumin concentration, which was excluded due to the high number of missing values. Continuous variables were recategorised into binary factors using the following threshold values: age $>60$ yrs, length of illness $>5$ days, temperature $<37.8^{\circ} \mathrm{C}$, cardiac frequency $>100$ beats $\cdot \mathrm{min}^{-1}$, respiratory rate $>30$ cycles $\cdot \mathrm{min}^{-1}$, systolic blood pressure $<90 \mathrm{mmHg}$, white blood cell count $>15,000 \mathrm{~mm}^{3}$, and neutrophil count $>12,000 \mathrm{~mm}^{3}$. In multivariable analysis, only five factors remained significantly associated with E/CPE: age, alcohol abuse, pleuritic pain, tachycardia and leukocytosis (table 2). The distribution of patients according to the number of predictive factors is shown in table 3 . The area under the ROC curve of this E/CPE prediction model was 0.71 (95\% CI 0.67-0.75). Assigning one point to each predictive factor, with a score of $\geqslant 2$ points as the cut-off, the model had a sensitivity of $85 \%$, specificity of $39 \%$, positive predictive value of $37 \%$ and negative predictive value of $87 \%$ for identifying E/CPE. The respective areas under the ROC curve of PSI and CRB-65 for predicting E/CPE were 0.56 (95\% CI 0.50-0.60) and 0.56 (95\% CI 0.52-0.60).

135 patients met criteria for empyema ( 80 had positive pleural fluid culture, 24 had macroscopic pus and 31 met both criteria) and 126 had CPE. A comparison between both subpopulations only showed mild differences in baseline characteristics and no differences in outcomes. Thus, patients with empyema had a longer length of disease prior to diagnosis (6 versus 3 days; $\mathrm{p}=0.016)$, a higher white blood cell counts (18,369 versus $15,135 \cdot \mathrm{mm}^{-3} ; \mathrm{p}=0.014$ ) and a lower haemotocrit level (37 versus $40 \% ; \mathrm{p}=0.002)$.

\section{Microorganisms}

After exclusion of any contaminating microorganism, a microbial aetiology was obtained in $2,667(57 \%)$ patients $(55 \%$ of patients without parapneumonic effusion, $60 \%$ of patients with UPE and $72 \%$ of patients with E/CPE), as shown in table 4 . S. pneumoniae was the most frequently identified bacterium in all subgroups of patients, being the cause of $30 \%$ of pneumonias without pleural effusion, $36 \%$ of pneumonias with UPE and $48 \%$ of pneumonias with E/CPE. A high incidence of other Grampositive cocci, particularly viridans streptcocci and anaerobes, were also isolated from patients with E/CPE. Conversely, in patients with E/CPE, no cases of pneumonia caused by atypical agents were found, with the exception of L. pneumophila. Polimicrobial infections were also more common in patients with $\mathrm{E}$ / CPE (9\% in patients with E/CPE versus $3 \%$ in patients with UPE or without pleural effusion). Most of cases of Gram-positive cocci and anaerobes were directly isolated from pleural fluid samples, as shown in table 5 .

\section{DISCUSSION}

In our study, $19 \%$ of patients with CAP developed pleural effusion and 6\% met criteria for E/CPE. Clinical and microbiological differences between patients without pleural effusion and patients with UPE were mild; although the presence of pleural fluid was associated with a longer hospitalisation. Conversely, the development of E/CPE was related to significant differences in many epidemiological, clinical and laboratory baseline characteristics. Specifically, alcoholism, younger age, pleuritic pain, tachycardia and leukocytosis were selected in a multivariable analysis as predictive factors of E/ CPE. Patients with E/CPE also showed a higher incidence of infections caused by Gram-positive cocci and anaerobes.

Chest radiograph has been demonstrated as a reliable technique to establish the presence of a significant amount of pleural fluid and constitutes the routine diagnostic test [10]. Small amounts of fluid can be detected by the use of thoracic ultrasound or computed tomography lung scan, but experiences suggesting a contributive role of these techniques to the management of patients are lacking. In addition, guideline recommendations are only based on chest radiographical findings [11, 12]. Consequently, we only used data provided by chest radiographs and found a rate of pleural effusion of $19 \%$, and a rate of $\mathrm{E} / \mathrm{CPE}$ of $6 \%$. These results are concordant with most recent, large epidemiological studies that report incidences of pleural effusion of $10-21 \%$ [13-15], and disagree with incidences of $40-60 \%$ referred to in some review papers $[2,8,16]$.

Thoracocentesis is now mandatory for patients with CAP and pleural effusion [2, 11]. A prediction model able to recognise patients with high risk for E/CPE could be useful to physicians in making decisions for treatment management of patients. In fact, thoracocentesis can be difficult or even risky in patients receiving anticoagulant therapy who are unable to collaborate or are prone to severe vagal reactions [17, 18]. A recent metaanalysis established that the risk of pneumothorax after thoracocentesis is $6 \%$ and that $34 \%$ of pneumothoraces require chest tube insertion [19].

AHMED et al. [20] compared 24 patients with empyema and 3,651 CAP patients without empyema. In the univariate analysis, the former were more likely to have a young age, a 
TABLE 1 Baseline characteristics of the three subgroups of patients with community-acquired pneumonia (CAP)

\begin{tabular}{|c|c|c|c|c|}
\hline Variables & Patients without pleural effusion & Patients with UPE & Patients with $\mathrm{E} / \mathrm{CPE}$ & p-values ${ }^{\#}$ \\
\hline Subjects $n$ & 3833 & 621 & 261 & \\
\hline Age yrs & $69(55-78)$ & $69(52-79)$ & $54(41-71)^{\#}$ & $<0.001$ \\
\hline Sex male & $2594(68)$ & $396(64)$ & $184(71)$ & 0.083 \\
\hline Underlying diseases & $2385(62)$ & $361(58)$ & $118(45)^{\#}$ & $<0.001$ \\
\hline Diabetes mellitus & $774(20)$ & $117(19)$ & $44(17)$ & 0.341 \\
\hline Heart failure & $808(21)$ & $140(23)$ & $26(10)^{\#}$ & $<0.001$ \\
\hline Chronic liver disease & $210(6)$ & $41(7)$ & $23(9)$ & 0.056 \\
\hline Chronic renal disease & $229(6)$ & $34(6)$ & $7(3)$ & 0.082 \\
\hline Neoplasm & $356(9)$ & $43(7)$ & $16(6)$ & 0.055 \\
\hline Cerebrovascular disease & $247(6)$ & $31(5)$ & $5(2)^{\#}$ & 0.006 \\
\hline Prior antibiotic therapy & $900(25)$ & $149(25)$ & $57(23)$ & 0.819 \\
\hline Pneumococcal vaccination & $443(16)$ & $58(13)$ & $14(8)^{\#}$ & 0.004 \\
\hline \multicolumn{5}{|l|}{ Clinical findings } \\
\hline Acute onset of disease & $1830(48)$ & $334(54)^{\#}$ & $125(48)$ & 0.040 \\
\hline Length of disease days & $3(2-6)^{\#}$ & $4(2-7)$ & $4(2-7)$ & $<0.001$ \\
\hline Fever & $3194(87)$ & $382(86)$ & $145(77)^{\#}$ & 0.001 \\
\hline Chills & $1688(45)$ & $290(47)$ & $110(43)$ & 0.418 \\
\hline Cough & $3080(82)$ & $532(86)^{\#}$ & $210(81)$ & 0.022 \\
\hline Expectoration & $2242(59)$ & $398(65)$ & $157(61)$ & 0.052 \\
\hline Pleuritic pain & $1474(39)^{\#}$ & $367(59)^{\#}$ & $201(77)^{\#}$ & $<0.001$ \\
\hline Signs of consolidation & $2959(79)$ & $524(85)$ & $200(78)^{\#}$ & 0.003 \\
\hline \multicolumn{5}{|l|}{ Vital signs } \\
\hline Oxygen saturation \% & $92(89-95)$ & $92(88-95)$ & $92(88-95)$ & 0.960 \\
\hline \multicolumn{5}{|l|}{ Laboratory results } \\
\hline C-reactive protein ${ }^{\S}$ & $248(162-359)$ & $260(159-330)$ & $278(187-278)$ & 0.992 \\
\hline Albumin $\mathrm{mg} \cdot \mathrm{dL}^{-1} f$ & $3.2(2.8-3.5)^{\#}$ & $3(2.8-3.4)^{\#}$ & $2.7(2.2-3)^{\#}$ & $<0.001$ \\
\hline Sodium mmol. $\mathrm{L}^{-1}$ & $137(134-140)$ & $137(134-140)$ & $137(133-140)$ & 0.928 \\
\hline Urea $\mathrm{mg} \cdot \mathrm{dL}^{-1} \# \#$ & $42(31-61)$ & $39(31-53)$ & $41(26-64)$ & 0.405 \\
\hline White cell count $\cdot \mathrm{mm}^{-3} \times 10^{3}$ & $13(9-18)$ & $13(9-18)$ & $16(12-22)^{\#}$ & $<0.001$ \\
\hline Neutrophil count $\cdot \mathrm{mm}^{-3} \times 10^{3}$ & $10(6-15)^{\#}$ & $12(7-16)^{\#}$ & $15(10-18)^{\#}$ & $<0.001$ \\
\hline Haematocrit \% & $39(35-43)^{\bullet}$ & $38(35-42)$ & $38(35-42)$ & 0.010 \\
\hline Arterial $\mathrm{pH}$ & $7.46(7.42-7.49){ }^{\bullet}$ & $7.45(7.42-7.49)$ & $7.45(7.41-7.49)$ & 0.979 \\
\hline $\mathrm{Pa}_{\mathrm{a}} \mathrm{O}_{2} \mathrm{mmHg}$ & $60(53-68)$ & $59(53-67)$ & $60(55-68)$ & 0.357 \\
\hline $\mathrm{Pa}, \mathrm{CO}_{2} \mathrm{mmHg}$ & $36(32-40)$ & $36(32-40)$ & $35(31-39)$ & 0.224 \\
\hline Positive blood cultures & $412(12)^{\#}$ & $94(16)^{\#}$ & $57(23)^{\#}$ & $<0.001$ \\
\hline \multicolumn{5}{|l|}{ PSI categories } \\
\hline Class 1 & $369(10)$ & $63(10)$ & $35(14)$ & 0.018 \\
\hline Class ॥ & $665(17)$ & $82(13)^{\#}$ & $49(19)$ & \\
\hline Class III & 829 (22) & $135(22)$ & $62(24)$ & \\
\hline Class IV & $1365(36)$ & $222(36)$ & $76(29)$ & \\
\hline Class V & $584(15)$ & $119(19)^{\#}$ & $38(15)$ & \\
\hline
\end{tabular}




\section{TABLE 1 Continued}

\begin{tabular}{|c|c|c|c|c|}
\hline Variables & Patients without pleural effusion & Patients with UPE & Patients with $\mathrm{E} / \mathrm{CPE}$ & p-values ${ }^{\#}$ \\
\hline \multicolumn{5}{|l|}{ CRB65 } \\
\hline 0 & $997(26)$ & $153(25)$ & $80(30)$ & 0.251 \\
\hline 1 & 1495 (39) & $252(41)$ & $108(41)$ & \\
\hline 2 & $1035(27)$ & $170(27)$ & $62(24)$ & \\
\hline 3 & $268(7)$ & $42(7)$ & $11(4)$ & \\
\hline 4 & $38(1)$ & $4(<1)$ & 0 & \\
\hline \multicolumn{5}{|l|}{ Outcomes } \\
\hline Mortality & $287(8)$ & $52(9)$ & $30(12)^{\#}$ & 0.048 \\
\hline Length of hospital stay & $7(5-11)^{\#}$ & $10(7-15)^{\#}$ & $17(12-26)^{\#}$ & $<0.001$ \\
\hline
\end{tabular}

Data are presented as median (25th-75th quartiles) or $n$ (\%), unless otherwise stated. UPE: uncomplicated parapneumonic effusion; E/CPE: empyema/complicated parapneumonic effusion; COPD: chronic obstructive pulmonary disease; $\mathrm{Pa}_{1} \mathrm{O}_{2}$ : arterial oxygen tension; $\mathrm{Pa}_{1} \mathrm{CO}_{2}$ : arterial carbon dioxide tension; $\mathrm{PSI}$ : pneumonia severity

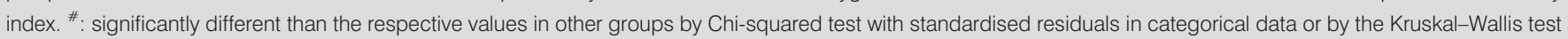
(post hoc analysis with the Mann-Whitney test) in continuous data; ${ }^{\bullet} \mathrm{n}=3,418$; $^{+}$: significantly different than the respective values in the group without pleural effusion;

s: $n=160 ;{ }^{f}: n=1,457 ;{ }^{\# \#}: n=1,116 ;{ }^{\cdots}$ : significantly different than the respective values in the uncomplicated pleural effusion group.

history of drug abuse, absence of history of chronic obstructive pulmonary disease or heart disease, fever, chills, pleuritic pain, tachycardia and high white blood cell and neutrophil counts [20]. More recently, CHALMERS et al. [21] conducted a multivariable analysis that compared 92 patients with E/CPE and 1,177 patients with CAP and identified seven clinical or laboratory findings associated with E/CPE: chronic alcohol abuse, intravenous drug use, absence of chronic obstructive lung disease, low albumin levels, low sodium levels, high Creactive protein and high platelet count.

Our results agree with most of the findings reported by these prior studies. Specifically, the multivariable analysis identified five predictive factors of E/CPE: young age ( $<60 \mathrm{yrs})$, alcoholism, pleuritic pain, tachycardia $\left(>100\right.$ beats $\left.\cdot \mathrm{min}^{-1}\right)$ and leukocytosis $\left(>15,000 \cdot \mathrm{mm}^{3}\right)$. Other previously described features associated with E/CPE lost their significance in the multivariable analysis $[19,20]$. Notably, the five selected risk factors are clinical or laboratory data that are easy to obtain and available at any hospital admission.

The selection of these predictor factors appears to be very reasonable. First, alcoholism has been recognised as an important predisposing factor for lung aspiration, facilitating the development of respiratory infections caused by microorganisms of the

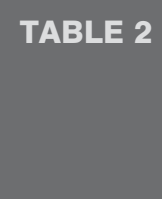

Multivariable analysis of predictive factors associated with empyema/complicated parapneumonic effusion among patients with community-acquired pneumonia and pleural effusion

\begin{tabular}{lcc} 
Variables & OR $\mathbf{( 9 5 \% ~ C l )}$ & p-value \\
\hline Age $<\mathbf{6 0}$ yrs & $1.74(1.13-2.67)$ & 0.012 \\
Alcoholism & $2.09(1.30-3.36)$ & 0.002 \\
Pleuritic pain & $1.92(1.27-2.89)$ & 0.002 \\
Tachycardia $>\mathbf{1 0 0}$ beats $\cdot \mathbf{m i n}^{-1}$ & $1.67(1.16-2.34)$ & 0.006 \\
Leukocytosis $>\mathbf{1 5 0 0 0} \cdot \mathbf{m m}^{-\mathbf{3}}$ & $2.30(1.61-3.29)$ & $<0.001$ \\
\hline
\end{tabular}

oropharyngeal flora, particularly anaerobes and oral streptococci, which are common agents of E/CPE [22, 23]. Secondly, investigators have found a reduced prevalence of clinical manifestations in older patients with CAP, suggesting age-related changes in the inflammatory response of the host to pathogens [24]. We can speculate on the transfer of this phenomenon to the pleural space. Thirdly, pleuritic pain is the most characteristic clinical consequence of the presence of inflammatory fluid in the pleural space; furthermore, in patients with CAP, pleuritic pain has been associated with infection by conventional bacteria and bacteraemia [25, 26]. Lastly, tachycardia and leukocytosis can be attributed to the major presence of typical and more aggressive microorganisms, as opposed to atypical agents. Alternatively, both abnormalities could be related to a more severe condition; in fact, the presence of pathogens in the pleural space is considered as an invasive infection going beyond the lung and increasing the inflammatory consequences of illness.

The description of the spectrum of causal microorganisms among the three subsets of patients represents another relevant contribution of our study, particularly because we achieved a

\section{TABLE 3 Patient distribution according to the number of predictive factors for empyema/complicated parapneumonic effusion (E/CPE) and the presence of pleural effusion}

\begin{tabular}{lccc}
$\begin{array}{l}\text { Predictive } \\
\text { factors n }\end{array}$ & $\begin{array}{c}\text { Patients without } \\
\text { pleural effusion }\end{array}$ & $\begin{array}{c}\text { Patients with } \\
\text { UPE }^{*}\end{array}$ & $\begin{array}{c}\text { Patients with } \\
\text { E/CPE+ }\end{array}$ \\
\hline $\mathbf{0}$ & $603(16)$ & $68(11)$ & $8(3)$ \\
$\mathbf{1}$ & $1279(33)$ & $177(29)$ & $30(11)$ \\
$\mathbf{2}$ & $1123(29)$ & $196(32)$ & $68(26)$ \\
$\mathbf{3}$ & $603(16)$ & $118(19)$ & $69(26)$ \\
$\mathbf{4}$ & $204(5)$ & $58(9)$ & $71(27)$ \\
$\mathbf{5}$ & $21(0.5)$ & $4(0.6)$ & $15(6)$ \\
\hline
\end{tabular}

Data are presented as number of patients (\%), unless otherwise stated. UPE: uncomplicated parapneumonic effusion. ${ }^{*}: n=3,833 ; ": n=621 ;{ }^{+}: n=261$ 
TABLE 4 Aetiological diagnosis in the three subgroups of patients with community-acquired pneumonia

\begin{tabular}{|c|c|c|c|}
\hline Aetiologies & Patients without pleural effusion ${ }^{\#}$ & Patients with UPE & Patients with $\mathrm{E} / \mathrm{CPE}^{+}$ \\
\hline Gram-positive cocci & $1221(32)$ & $235(38)$ & $168(64)$ \\
\hline Streptococcus pneumoniae & $1178(388 / 790)$ & $224(75 / 149)$ & $124(102 / 22)$ \\
\hline Streptococcus viridans & $20(20 / 0)$ & $6(6 / 0)$ & $34(34 / 0)$ \\
\hline Other & $3(2 / 1)$ & $1(1 / 0)$ & $4(4 / 0)$ \\
\hline Anaerobes & 0 & 0 & $25(10)$ \\
\hline Haemophilus influenzae & $181(17 / 164)$ & $25(6 / 19)$ & $5(3 / 2)$ \\
\hline Moraxella catarrhalis & $23(2 / 21)$ & $8(0 / 8)$ & 0 \\
\hline Pseudomonas aeruginosa & $38(26 / 12)$ & $5(4 / 1)$ & $2(1 / 1)$ \\
\hline Escherichia coli & $18(13 / 5)$ & $3(2 / 1)$ & $2(2 / 0)$ \\
\hline Klebsiella spp. & $5(2 / 3)$ & $3(2 / 1)$ & $3(2 / 1)$ \\
\hline Other & $15(9 / 6)$ & $3(2 / 1)$ & $3(3 / 0)$ \\
\hline Chlamydophila psitacii & $19(19 / 0)$ & $1(1 / 0)$ & 0 \\
\hline Coxiella burnetti & $56(56 / 0)$ & $8(8 / 0)$ & 0 \\
\hline Virus & $50(50 / 0)$ & $9(9 / 0)$ & 0 \\
\hline Other pathogens & 11 & 2 & 1 \\
\hline Mixed infections & $101(3)$ & $19(3)$ & $23(9)$ \\
\hline Unknown aetiology & $1726(45)$ & $249(40)$ & $73(28)$ \\
\hline
\end{tabular}

Data for subgroups of pathogens are presented as $n(\%)$; data for microorganisms are presented as total (definitive diagnosis/probable diagnosis). UPE: uncomplicated pleural effusion; E/CPE: empyema/complicated parapneumonic effusion. ${ }^{\#}: n=3,833 ;{ }^{\bullet}: n=621 ;{ }^{+}: n=261$.

high percentage of known aetiologies. There were no differences between patients without pleural effusion and patients with UPE. However, for patients with E/CPE, Gram-positive cocci and anaerobes were the predominant agents, and $S$. pneumoniae was the pathogen in nearly $50 \%$ of cases. It is also important to stress that the incidence of Gram-negative bacilli

\begin{tabular}{|cc|}
\hline TABLE 5 & $\begin{array}{l}\text { Microorganisms isolated from pleural fluid in } \\
\text { patients with community-acquired pneumonia } \\
\text { and empyema/complicated parapneumonic } \\
\text { effusion }\end{array}$ \\
Gram-positive cocci & $111(76)$ \\
Streptococcus pneumoniae & 77 \\
Staphylococcus aureus & 5 \\
Streptococcus viridans & 26 \\
Other & 3 \\
Anaerobes & $25(17)$ \\
Gram-negative bacilli & $8(5)$ \\
Haemophilus influenzae & 2 \\
Escherichia coli & 2 \\
Klebsiella spp. & 1 \\
Other & 3 \\
Atypical agents & $2(1)$ \\
Legionella pneumophila & 2 \\
Mixed infections & $20(8)$ \\
\hline
\end{tabular}

Data are presented as $n(\%)$ or $n$ appeared not to be related to pleural effusion findings and that, except for some cases of Legionella, atypical agents did not cause E/CPE. Empirical treatments should consider these aetiological particularities.

In a therapeutic study on patients with empyema, MASKELL et al. [27] showed similar results on the relative prevalence of isolated pathogens, although S. pneumoniae had only a secondary role. We believe there may be several reasons for this discrepancy. First, populations in both studies were different; thus, patients included in the study by MASKELL et al. [27] had a very long duration of symptoms prior to diagnosis, almost all met criteria for empyema, and $\sim 20 \%$ had primary empyema or nosocomial infection. In addition, the microbiological evaluation of patients was limited to blood and pleural fluid cultures. Other prior investigations on the aetiology of E/CPE were limited by the small number of cases with aetiological diagnosis and the reduced number of microbiological techniques employed [20, 21].

The presence of pleural fluid was associated with a worse prognosis. The duration of hospitalisation increased in patients with UPE, and both mortality and duration of hospitalisation were increased in patients with E/CPE. These findings have been described by others; in fact, pleural effusion was already included in the score for predicting the mortality risk in the PSI $[4,6,28]$.

We should recognise some limitations in our study. First, baseline characteristics were collected at admission; however, in some cases, the presence of pleural effusion was detected later, during admission or even after discharge. We can suspect that a 
small amount of pleural fluid was already present at admission, but this constitutes a potential limitation in the evaluation of results. Secondly, deficiencies in the collection, transport or processing of samples, particularly in relation to the isolation of anaerobes, probably influenced the proportion of cases with this aetiology. Thirdly, the predictive role of some variables, significantly associated with empyema in other studies (albumin, C-reactive protein or platelet count), could not be avaluated because they were not accurately collected. Finally, it should be noted that all patients with pneumonia were enrolled in the study; this population may occasionally include patients with CAP in whom pleural effusion was caused by underlying diseases or other associated conditions.

In summary, we found that the presence of UPE had only mild prognostic consequences; however, the development of E/CPE characterised a subgroup of somewhat predictable patients with significant baseline differences and microbiological particularities. Our results may be useful to the clinical management and the selection of treatment for patients with CAP.

\section{STATEMENT OF INTEREST}

None declared.

\section{ACKNOWLEDGEMENTS}

This work was supported by Ciber de Enfermedades Respiratorias (CIBERES CB06/06/0028) from the Instituto de Salud Carlos III, Madrid, Spain, and the European Development Regional Fund "A way to achieve Europe" ERDF, Spanish Network for the Research in Infectious Diseases (REIPI RD06/"'8).

\section{REFERENCES}

1 Porcel JM. Pearls and myths in pleural fluid analysis. Respirology 2011; 16: 44-52.

2 Colice GL, Curtis A, Deslauriers J, et al. Medical and surgical treatment of parapneumonic effusions. An evidence-based guideline. Chest 2000; 18: 1158-1171.

3 Sahn SA. Use of fibrinolytic agents in the management of complicated parapneumonic effusions and empyemas. Thorax 1998; 53: 65-72.

4 Lindstrom ST, Kolbe J. Community-acquired parapneumonic thoracic empyema: predictors of outcome. Respirology 1999; 4: 173-179.

5 Marik PE. Aspiration pneumonitis and aspiration pneumonia. $N$ Engl J Med 2001; 344: 665-671.

6 Fine MJ, Auble TE, Yealy DM, et al. A prediction rule to identify low-risk patients with community-acquired pneumonia. $N$ Engl J Med 1997; 336: 243-250.

7 Lim WS, van der Eerden MM, Laing R, et al. Defining communityacquired pneumonia severity on presentation to hospital: an international derivation and validation study. Thorax 2003; 58: 377-382.

8 Light RW, Girard WM, Jenkinson SG, et al. Parapneumonic effusions. Am J Med 1980; 69: 507-512.

9 Hosmer DW, Lemeshow S. Model-building strategies and methods for logistic regression. In: Hosmer DW, Lemeshow S, eds.
Applied Logistic Regression. 2nd Edn. New York, John Wiley and Sons, 2000; pp. 91-142.

10 Boersma WG, Daniels JMA, Löwenberg A, et al. Reliability of radiographic findings and the relation to etiologic agents in community-acquired pneumonia. Respir Med 2006; 100: 926-932.

11 Mandell LA, Wunderink RG, Anzueto A, et al. Infectious Diseases Society of America/American Thoracic Society consensus guidelines on the management of community-acquired pneumonia in adults. Clin Infect Dis 2007; 44: 27-72.

12 Lim WS, Baudouin SV, George RC, et al. British Thoracic Society guidelines for the management of community acquired pneumonia in adults: update 2009. Thorax 2009; 64: 1-55.

13 Phua J, See KC, Chan YH, et al. Validation and clinical implications of the IDSA/ATS minor criteria for severe community-acquired pneumonia. Thorax 2009; 64: 598-603.

14 Viegi G, Pistelli R, Cazzola M, et al. Epidemiological survey on incidence and treatment of community-acquired pneumonia in Italy. Respir Med 2006; 100: 46-55.

15 Luna CM, Famiglietti A, Absi R, et al. Community-acquired pneumonia. Etiology, epidemiology, and outcome at a teaching hospital in Argentina. Chest 2000; 118: 1344-1354.

16 Woodhead M, Blasi F, Ewig S, et al. Guidelines for the management of adult lower respiratory tract infections. Eur Respir J 2005; 26: 1136-1180.

17 Roth M, Hal Cragun LTC, Grathwohl CPT. Complications associated with thoracocentesis. Arch Intern Med 1991; 151: 2095-2096.

18 Collins TR, Sahn SA. Thoracocentesis. Clinical value, complications, technical problems, and patient experience. Chest 1987; 91: $817-822$.

19 Gordon CE, Feller-Kopman D, Balk EM, et al. Pneumothorax following thoracentesis. A systematic review and meta-analysis. Arch Intern Med 2010; 170: 332-339.

20 Ahmed RA, Marrie TJ, Huang JQ. Thoracic empyema in patients with community-acquired pneumonia. Am J Med 2006; 119: 877-883.

21 Chalmers JD, Singanayagam A, Murray MP, et al. Risk factors for complicated parapneumonic effusion and empyema on presentation to hospital with community-acquired pneumonia. Thorax 2009; 64: 592-597.

22 Barlett JG. Anaerobic bacterial infections of the lung and pleural space. Clin Infect Dis 1993; 16: 248-255.

23 Alfagame I, Muñoz F, Pena N, et al. Empyema of the thorax in adults, etiology, microbiologic findings and management. Chest 1993; 103: 839-843.

24 Metlay JP, Schulz R, Li YH, et al. Influence of age on symptoms at presentation in patients with community-acquired pneumonia. Arch Intern Med 1997; 157: 1453-1459.

25 Kauppinen MT, Saikku P, Kujala P, et al. Clinical picture of community-acquired Chlamydia pneumoniae pneumonia requiring hospital treatment: a comparison between chlamydial and pneumococcal pneumonia. Thorax 1996; 51: 185-189.

26 Falguera M, Trujillano J, Caro S, et al. A prediction rule for estimating the risk of bacteremia in patients with communityacquired pneumonia. Clin Infect Dis 2009; 49: 409-416.

27 Maskell NA, Batt S, Hedley EL, et al. The bacteriology of pleural infection by genetic and standard methods and its mortality significance. Am J Respir Crit Care Med 2006; 174: 817-823.

28 García-Vidal C, Carratalà J, Díaz V, et al. Factors associated with prolonged hospital stay in community-acquired pneumonia. Enferm Infecc Microbiol Clin 2009; 27: 160-164. 